\section{DGI-058 RESULTS OF USING TOLVAPTAN}

doi:10.1136/ejhpharm-2013-000276.324

${ }^{1} \mathrm{MD}$ Cantudo Cuenca, 'A Acuña Vega, 'MB Dominguez Aguado, 'M Vargas Lorenzo, ${ }^{1} \mathrm{C}$ Del Moral Alcazar, 'R Saldaña Soria, ${ }^{2} \mathrm{R}$ Cantudo Cuenca, 'MJ Barbero Hernandez, IF Horno Ureña. 'Complejo Hospitalario Jaén, Pharmcy Service, Jaén, Spain; ${ }^{2}$ Area Hospitalaria Valme, Pharmcy Service, Sevilla, Spain

Background Tolvaptan is the first oral antagonist of the vasopres$\sin \mathrm{V} 2$ receptor. It is indicated in adult patients with hyponatraemia secondary to syndrome of inappropriate antidiuretic hormone hypersecretion (SIADH).

Purpose To evaluate the use of tolvaptan in a tertiary hospital. Materials and Methods An observational study was conducted on patients treated with tolvaptan from January 2012 to September 2012. Data was collected from the review of medical histories, lab tests and dispensing records. A data collection sheet was designed on which were recorded: diagnosis related to hyponatraemia, age, gender, dose, clinical department that prescribed it, serum sodium when the treatment with tolvaptan was initiated, evolution and possible side effects.

Results 6 patients $(50 \%$ male) received tolvaptan in the study period. Average age was 72.53 years. The clinical department that wrote the prescription was Internal Medicine in five cases and Oncology in the other one. The background pathology was lung cancer in two cases, heart failure in two cases, idiopathic SIADH in one case and only one case of SIADH. The average serum sodium concentration pre-treatment was 113 (101-120) mg/dl. The dose usually used was $15 \mathrm{mg} /$ day, although one patient took $30 \mathrm{mg} /$ day. The average length of treatment was 123 (30-270) days. Only one patient discontinued treatment due to gastrointestinal side effects. One terminal cancer patient and an 85-year-old patient died. The average cost-day per patient was $€ 65.75$.

Conclusions Our results agree with the tolvaptan clinical trials, that it appears to be safe and effective in the treatment of hyponatraemia refractory to other treatments. The high cost of the treatment and the limited experience in its use required strict control over its administration.

No conflict of interest.

\section{DGI-059 SAFETY OF ANTI-EPIDERMAL GROWTH FACTOR RECEPTOR AGENTS: CETUXIMAB AND PANITUMUMAB}

doi:10.1136/ejhpharm-2013-000276.325

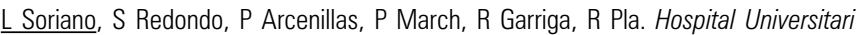
Mútua de Terrassa, Pharmacy, Terrassa, Spain

Background A recently-published meta-analysis describes the risk of thromboembolic events (TEs) associated with anti-growth factor receptors such as cetuximab and panitumumab.

Purpose To describe the frequency of TEs related to cetuximab and panitumumab use. Likewise, to detail adverse reactions (ARs) and their severity.

Materials and Methods Retrospective descriptive study in a 500bed university hospital performed from January 2010 to September 2012. All patients who had been treated with cetuximab or panitumumab were reviewed. In a database we recorded: sex, age, underlying disease, drug, dose reduction if it was necessary, number of cycles administered, ARs and degree of severity according to Common Toxicity Criteria. The information was extracted from patients' medical records and from pharmacy service records.

Results Twenty-four patients were included, 12 were men. Mean sample age was 61 years. The main underlying disease was colorectal cancer with liver and lung metastases $(41.2 \%)$. Mean duration of treatment was 10.7 cycles/patient. All patients received cetuximab in combination regimens with fluoropyrimidines, platinum and irinotecan. Four patients were treated with panitumumab. ARs appeared in $95.8 \%$ of the sample. There were 153 ARs, $88.9 \%$ during treatment with cetuximab. (Table 1). Two cases of deep vein thrombosis (DVT) during treatment with cetuximab were reported; none with panitumumab. Grade 1 toxicity represented $44.5 \%$ of all ARs, $40.5 \%$ were grade $2,13.7 \%$ grade 3 and $1.3 \%$ grade 4 . Due to ARs, three patients required dosage reduction, all related to cetuximab schedules.

Conclusions Two cases of DVT were reported in patients treated with different cetuximab chemotherapy schedules. It is difficult to establish a relationship between ARs and the drugs used. Further studies are needed to clarify the association of TE and cetuximab. The rest of AR founded, are described in the product information. It is necessary a higher foresight to establish preventive measures to avoid or reduce AR toxicity.

\section{Abstract DGI-059 Table 1}

\begin{tabular}{ll}
\hline AR & \% of patients \\
\hline Rash & 79.2 \\
Paresthesia & 66.7 \\
Transaminases & 54.2 \\
Asthenia & 45.8 \\
Diarrhoea & 37.5 \\
Neutropenia & 29.2 \\
Anaemia & 25.0 \\
Tricomegalia & 25.0 \\
\hline
\end{tabular}

No conflict of interest.

\section{DGI-060 SAFETY OF INTRAVENOUS TREATMENT OF BREAST CANCER: INTERACTION WITH CHRONIC MEDICINES}

doi:10.1136/ejhpharm-2013-000276.326

M Sebastián-Aldeanueva, MJ Agustín, I Villar, YM Alonso-Triana, H Aznárez, R Huarte, P Palomo, R Abad. Servet, Pharmacy, Zaragoza, Spain

Background Pharmacists may play an important role in the prevention of potential drug interactions (PDIs).

Purpose To investigate PDIs among intravenous cytotoxic drugs and medicines for comorbid illnesses in breast cancer patients, according to the interaction mechanism, its clinical significance and the published literature.

Materials and Methods Treatments for breast cancer patients were analysed in a retrospective study over a month. Data were collected from pharmacy oncology software (Oncowin) and the Primary care Prescription Data-Base (OMI-AP). Interactions were checked with Lexi-Comp Online.

Results 73 women were treated with intravenous cytotoxic drugs in November 2011. Mean age was $57 \pm 13$ years. Only 40 women were recorded in the Primary Care prescription database, and 3 of them did not receive concomitant treatment during that month. There were 10 different chemotherapy schemes involving 7 antineoplastic drugs. Comorbid chronic diseases were treated with 89 different drugs; antihypertensives, NSAIDs, benzodiazepines and antimicrobials were the most widely used drugs. 7 cases of PDIs were found, comprising 5 different interactions: cyclophosphamide/paroxetine (2), paclitaxel/diltiazem (1), docetaxel/trazodone (1), paclitaxel/atorvastatin (2), paclitaxel/ketoconazole (1). These interactions were detected in 6 patients (15\% of patients with OMIAP data). In one patient 2 PDIs were observed: cyclophosphamide/ paroxetine and docetaxel/trazodone. All the PDIs detected were pharmacokinetic interactions. None of the PDIs detected had clinical relevance according to the scientific literature.

Conclusions PDIs may occur among drugs for chronic diseases and chemotherapy in breast cancer patients. These data are consistent with previous reports in which PDIs were observed in 19\% of 
cancer patients. Most relevant interactions described are paclitaxel with antiepileptics, docetaxel with ketoconazole or cyclophosphamide with benzodiazepines. No clinically relevant interactions were found in our patients. Patients with comorbidities on multiple drug therapy (in addition to the drugs used for cancer treatment) would most benefit from pharmaceutical care.

No conflict of interest.

\section{DGI-061 SAFETY OF TRIPLE TREATMENT IN CHRONIC HEPATITIS C}

doi:10.1136/ejhpharm-2013-000276.327

P Marrero, L Lorente, S Valero, I Gil, E Monte, J Ruiz, J Reig, JL Poveda. Hospital Universitari i Politecnic La Fe, Hospital Pharmacy, Valencia, Spain

Background Efficacy of chronic hepatitis C genotype 1 treatment has been improved with protease inhibitors (PIs) telaprevir and boceprevir. However, triple therapy (PI, peginterferon alfa and ribavirin) has increased the number, type and severity of adverse events.

Purpose To assess the safety of triple therapy in the first 12 weeks of treatment with telaprevir and boceprevir used for chronic hepatitis $\mathrm{C}$ treatment in clinical practise.

Materials and Methods Between March and September 2012, all patients treated with telaprevir and boceprevir receiving medicines in the outpatient pharmaceutical care unit of a tertiary hospital were interviewed. Adverse events were collected in a predefined questionnaire. Anaemia, neutropenia and thrombocytopenia were also included as adverse effects if the patient had been treated for any of them. Interviews were conducted during the medicines dispensing (monthly).

Results Fifty-one patients with triple therapy were interviewed; 34 of them were treated with telaprevir and 17 with boceprevir. All patients had at least one adverse event on any of the visits. Globally, the most frequent adverse events were tiredness (84.3\%), digestive disorders $(70.6 \%)$, dermatological disorders $(64.7 \%)$ and influenzalike syndrome (62.7\%). Patients being treated with telaprevir mainly suffered from tiredness (85.3\%) and dermatological disorders (70.6\%). However, tiredness (82.4\%) and mood disorders $(70.6 \%)$ were the most usual adverse events in patients being treated with boceprevir. The frequencies of other side effects are listed in Table 1. Conclusions Efficacy in the first 12 weeks of triple therapy results in a high frequency of adverse events. Information on possible side effects and how to prevent or treat them is important for patients. Since PIs have only recently come onto the market, it is also important to communicate and record any new adverse events not identified in clinical trials.

No conflict of interest.

\section{DGI-062 SORAFENIB, SUNITINIB AND EVEROLIMUS IN METASTATIC RENAL CELL CARCINOMA: EFFICACY AND SAFETY}

doi:10.1136/ejhpharm-2013-000276.328

I Ormazabal Goicoechea, M Polanco Paz, P Sanmartin Fenollera, M Perez Encinas. Hospital Universitario Fundación Alcorcón, Pharmacy, Madrid, Spain

Background Tyrosine kinase inhibitors (TKIs) and m-TOR inhibitors ( $\mathrm{m}$-TORIs) have demonstrated clinical efficacy in patients with advanced renal cell carcinoma (aRCC).
Abstract DGI-061 Table 1

\begin{tabular}{lll}
\hline Adverse event & Telaprevir (\%) & Boceprevir (\%) \\
\hline Influenza-like illness & 61.8 & 64.7 \\
Tiredness & 85.3 & 82.4 \\
Mood disorders & 32.4 & 70.6 \\
Digestive disorders & 67.7 & 76.5 \\
Dermatological disorders & 70.6 & 52.9 \\
Hair lost & 5.9 & 17.7 \\
Non-productive cough & 8.8 & 29.4 \\
Itchy eyes & 0.0 & 5.9 \\
Oral disorders & 32.4 & 33.3 \\
Haemorrhoids & 64.7 & 0.0 \\
Tachycardia & 2.9 & 23.5 \\
Decreased libido & 2.9 & 11.8 \\
Oedema & 11.8 & 11.8 \\
Anaemia & 55.9 & 47.6 \\
Neutropenia & 17.7 & 11.8 \\
Thrombocytopenia & 14.7 & 5.9 \\
\hline
\end{tabular}

Purpose To describe one centre's experience with the use of TKIs and an oral $\mathrm{m}$-TORI in patients with aRCC.

Materials and Methods Retrospective observational study of patients with aRCC treated with TKIs (sorafenib, sunitinib) and an m-TORI (everolimus) from March 2007-May 2012. Variables: demographics, initial ECOG, line number, duration (TT) and reason for stopping treatment, best response (partial response (PR), stable disease (SD), progression) according to clinical and radiological criteria; progression-free survival (PFS) and overall survival (OS) in weeks (w) and toxicity.

Results Of the 22 patients studied $81.8 \%$ were male with an average age of 65.77 years (SD: 11.76): 5 treated with sorafenib, 13 with sunitinib and 4 with everolimus.

Reasons for discontinuing were: 40\% (2/5), 46.15\% (6/13) and $75 \%$ (3/4) progression/clinical worsening; 40\% (2/5), 15.38\% (2/13) and $25 \%(1 / 4)$ toxicity; and 20\% (1/5), 15.38\% (2/13) and $0 \%$ death, for sorafenib, sunitinib and everolimus respectively. Response rates were (except the 5 patients who stopped too early): sorafenib $100 \%$ SD (2/2); sunitinib 25\% SD (3/12), 58.33\% PR (7/12) and $16.6 \%$ progression (2/12) and everolimus $100 \%$ progression (3/3).

Treatment-related adverse events: sorafenib $60 \%$ asthenia and 40\% rash; sunitinib: $53.85 \%$ rash, $46.15 \%$ diarrhoea and $38.46 \%$ neutropenia, mucositis and asthenia, and everolimus: $75 \%$ hypercholesterolemia, $50 \%$ hypertriglyceridemia and $25 \%$ pneumonitis.

Conclusions In our study, median OS was lower than those obtained in pivotal trials, instead, median PFS was higher, except everolimus. Regarding safety, sorafenib had similar toxicity; sunitinib had higher rates of hand-foot syndrome and everolimus had higher rates of hypercholesterolemia. However, the small number of patients limits our conclusions.

No conflict of interest.

\section{DGI-063 STUDY OF THE USE AND EFFECTIVENESS OF DAPTOMYCIN IN A TERTIARY HOSPITAL}

doi:10.1136/ejhpharm-2013-000276.329

M Bullejos Molina, I Rodriguez Pedrosa, J Nazco Casariego, I Gonzalez Perera, J Gonzalez Garcia. Hospital Universitario de Canarias, Servicio De Farmacia, La Laguna, Spain

Abstract DGI-062 Table 1

\begin{tabular}{|c|c|c|c|c|c|c|c|c|c|c|c|}
\hline & \multicolumn{4}{|c|}{ ECOG } & \multicolumn{4}{|c|}{ No. metastatic sites } & \multicolumn{2}{|c|}{ Line number } & \multirow[t]{2}{*}{ Median TT (w) } \\
\hline & 0 & 1 & 2 & No data & 1 & 2 & 3 & Common sites & 1 & $\geq 2$ & \\
\hline SORAFENIB & & $3 / 5$ & & $2 / 5$ & $2 / 5$ & $3 / 5$ & & $40 \%$ Lung $40 \%$ Bones & $4 / 5$ & $1 / 5$ & 5 (IOR: 2-49.5) \\
\hline EVEROLIMUS & $1 / 4$ & $3 / 4$ & & & $2 / 4$ & $1 / 4$ & $1 / 4$ & $50 \%$ Liver & $0 / 4$ & $4 / 4$ & 10 (IOR: 8-12) \\
\hline
\end{tabular}

